\title{
AC Magnetic sensor to measure mega-amps current and kilo-tesla magnetic fields up to gigahert frequencies
}

\author{
J. L. Lopez Segura ${ }^{1}$, N. Urgoiti Moinot ${ }^{1}$, A. Pal ${ }^{1}$ \\ ${ }^{1}$ Advanced Ignition SL C/francisco Medina Y Mendoza, Parcela 1, Poligono 19171 - (Cabanillas Del Campo) - \\ Guadalajara, Spain
}

(Received: 14. July 2020, Accepted: 11. August 2020, Published online: 12. August 2020

An AC magnetic sensor is presented for measuring the high speed and high strength magnetic field generated in Z-pinch fusion machines. The proposed magnetic sensor provides the measurement of magnetic fields across a broadband frequency range, as example, a magnetic probe to measure magnetic fields from $159 \mathrm{~Hz}$ to $4.2 \mathrm{GHz}$ is presented. Measurements are also compared with LTspice ${ }^{\circledR}$ simulations. The magnetic sensors are installed in a Pulsotron-3 Z-pinch machine and measured performance of the sensor are presented. This sensor also can be used to check ignition conditions of the Z-Pinch by measuring the magnetic field generated by the output streams of large number of reacted alpha particles. The equations for measuring nonstationary magnetic field due to rapidly varying electric currents and LTspice ${ }^{\circledR}$ simulation files are provided to help the engineers to design, build and install this kind of sensors.

(C) G-Labs 2020

(DOI: 10.31281/jtsp.v1i1.13)

jlopez@advancedignition.eu

\section{Introduction}

Magnetic field measurements are extensively used in the field of magnetically confined fusion [1-3]. These magnetic diagnostics [4] are required for understanding plasma behaviour. Existing magnetic diagnostics technique use inductive coils and loops for magnetic sensors [5-6]. These sensors are placed outside the plasma boundary but within the plasma's external magnetic fields. The changing magnetic field in the sensor induces an Electro Motive Force (EMF) which is integrated over time to determine the desired plasma property [7]. The state-of-the-art magnetic sensors based on the Hall effect [8] measures magnetic fields up to 0.2 tesla across a $20 \mathrm{kHz}$ bandwidth. Therefore, these sensors are completely out of range for Z-pinches that can reach more than 10 kilotesla and pulse width of 100-2000 nanoseconds. In addition, these sensors are not suitable to be used in plasma measurement due to poor signal to noise ratio and a narrow bandwidth. Moreover, high frequency magnetic sensors for measuring magnetic fields up to gigahertz frequencies are required to be in near proximity of the plasma [9]. After several tests carried out using Pulsotron-2A and Pulsotron-3 it is recommended that it is necessary to install several magnetic sensors in an array that can measure several parameters of the discharging energy through a plasma in Z-pinches. Also, it is needed a small and accurate magnetic sensor to be immersed in plasma to measure its parameters as skin depth and plasma impedance that can be useful to study plasma properties and help make magnetic confinement fusion.

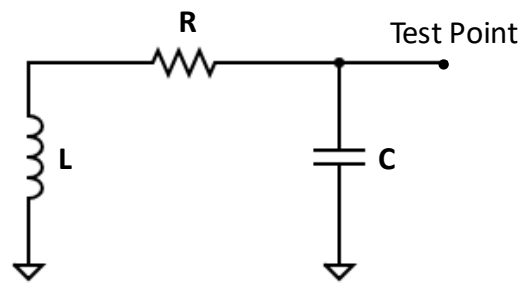

Figure 1: Configuration of the magnetic field probe. 


\section{Parameters to be measured}

The proposed magnetic sensor arrays can be used to measure directly the magnetic field, discharged current and Time of Flight (TOF). In addition, these sensors can be used to measure the plasma energy density (by measure plasma dimension), pressure (explained later), plasma double layer measurements (by using the TOF technique explained later, that detects the passing of both plasma layers through the sensors with inverted magnetic field) and several other parameters. These parameters can be used in Nuclear Fusion not only to improve Ignition Conditions but also to design energy recovery components to extract electric energy directly from the reacted particles.

\section{Description of the magnetic field probe}

Inductive sensors [10] are widely used to measure magnetic fields and related quantities. These sensors are linear up to the range of gigahertz frequencies and are easily calibrated precisely by install the sensor inductor in the centre of a loop and parallel to the loop axis (it is explained later the non-stationary magnetic field of current loops). Fig. 1 shows the configuration of the magnetic field probe of the Mag-4 sensor. It consists of an inductor $L$, a resistors $R$ and $a$ capacitor $C$. The inductor $L$ is a coil that is immersed in the magnetic field. The resistor $\mathrm{R}$ and the capacitor $C$ are used to integrate the input signal that was derivate by the inductor coil L. Consequently, the sensor is linear across its frequency limit due to the passive components. The magnetic field probe is simulated using SPICE simulation software LTspice [11]. It was found that the voltage measured at test point is directly proportional to the induced magnetic field at the coil. Since the magnetic field is proportional to the injected current at the plasma surface, the current flowing through the plasma can also be calculated.

If the sensor probes have large area and longer length, it catches several magnetic fields. Therefore, sensor probes should be small in order to avoid error due to different magnetic field pass through the sensor. Furthermore, it must avoid the use of ferrites that modify the magnetic field shape. Thus, the proposed magnetic sensor should use very compact inductors. Fig. 2 shows the schematic layout and fabricated prototype of the magnetic sensor.

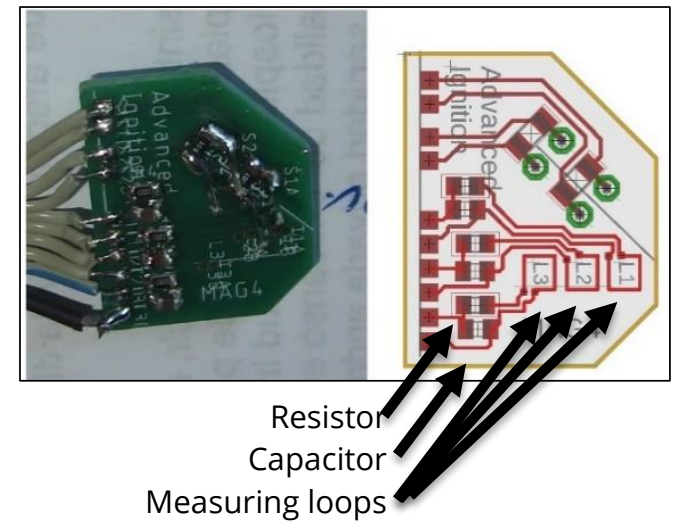

Figure 2: Image and PCB diagram of the MAG-4 sensor, Advanced Ignition SL.

These sensors are installed in Pulstron-3 Z-pinch machine for analysing its performance. Fig. 3 shows the mounting structure of the mag- 4 sensor in Pulsotron-3.

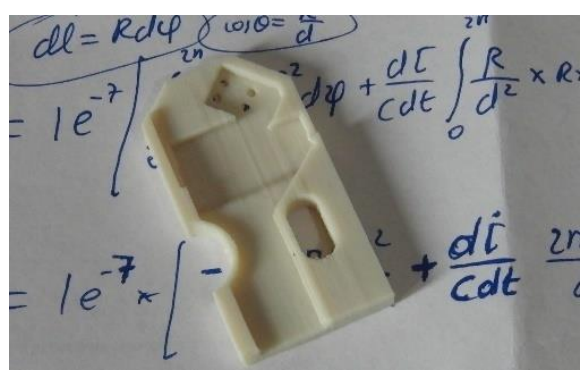

Figure 3: Mounting structure of the Pulsotron-3 Mag-4 sensor

\section{Voltage and bandwidth calculation}

At low frequency the coil behaves as similar as a short circuit and the probe provides the output signal of a RC low-pass filter. The $3 \mathrm{~dB}$ cutoff frequency of the low pass filter can be calculated as

$$
F_{L P}=\frac{1}{2 \pi R C}
$$

On the other hand, at high frequencies the capacitor becomes a short circuit and the output signal corresponds to a RL high-Pass filter with a $3 \mathrm{~dB}$ cut off frequency

$$
F_{H P}=\frac{R}{2 \pi L}
$$

The operating centre frequency of the magnetic probe can be calculated by taking the square root of the product of both frequencies and given as

$$
F_{C}=\frac{1}{\sqrt{4 \pi^{2} L C}}
$$


Fig. 4 shows the schematic diagram of the magnetic probe in LTspice. Fig. 5 shows the simulated response of the magnetic sensors when $\mathrm{L}=100 \mathrm{nH}, \mathrm{R}=100 \Omega$ and $\mathrm{C}=10 \mu \mathrm{F}$. A high-pass cutoff frequency of $159.15 \mathrm{~Hz}$ and a low-pass cutoff frequency of $159 \mathrm{MHz}$ was observed in the simulation.

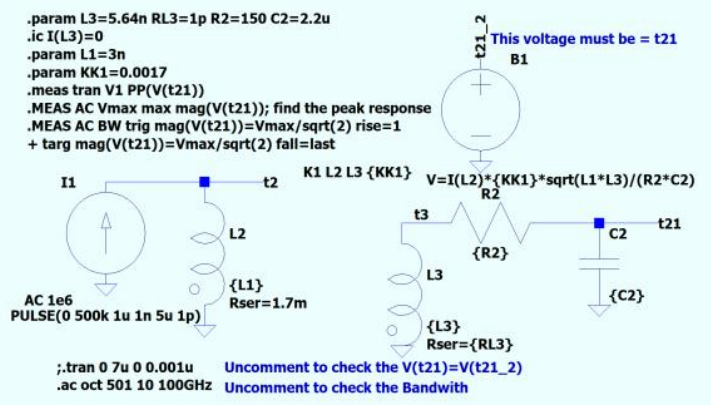

Figure 2: AC Simulation setup of the magnetic sensor in LTspice.

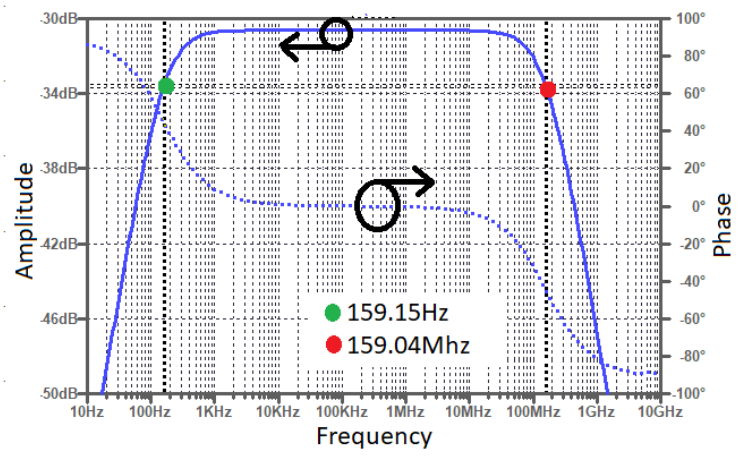

Figure 3: Low frequency magnetic sensor.

In order to extend the upper frequency range over a Gigahertz or beyond, the magnetic probe was simulated with a coil inductance $L=5.64 \mathrm{nH}$ and resistance $\mathrm{R}=150 \Omega$. The capacitor was reduced to $2.2 \mu \mathrm{F}$ to increase the output voltage level at test point. Consequently, the magnetic probe operates at higher frequency and it was found that the high-pass cutoff frequency is $482.288 \mathrm{~Hz}$ and the low-pass cutoff frequency is 4.23285G Hz as shown in Fig. 6.

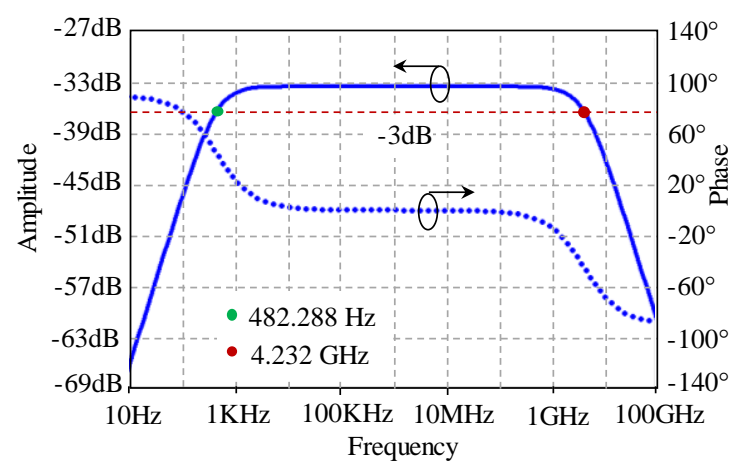

Figure 4: High frequency magnetic sensor.

\section{Equations used to measure plasma current}

According to Ampère's Law [12], the magnetic field $(B)$ around a current $(I)$ passing through a plasma is:

$$
\oint \vec{B} \cdot \overrightarrow{d l}=\mu_{0} I
$$

Where, $\mu_{0}$ is the magnetic permeability of free space. The current on the surface of a cylindrical plasma can be calculated from Eq. 4 and given as

$$
B_{\theta}=\frac{\mu_{0} I}{2 \pi r}
$$

Where, $r$ is the radius of the plasma cylinder. It can be seen from Eq. 5 that the magnetic field inside the plasma is 0 .

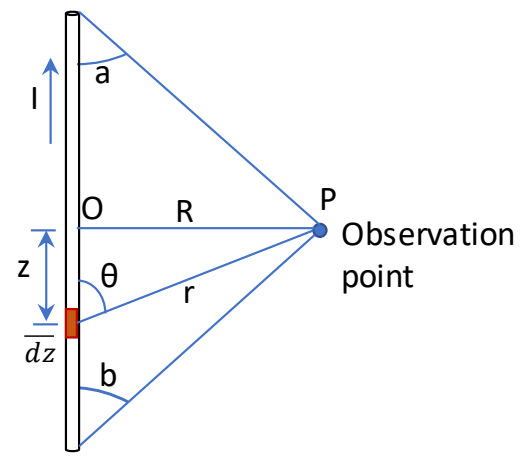

Figure 5: A segment of thin cylindrical currentcarrying wire or plasma.

Fig. 7 shows a segment thin straight cylindrical wire that carries a current \%. The observation point $P$ is located a distance $R$ from the currentcarrying wire. The independent variable $\theta$ has limits $a$ and $(n-b)$. If the plasma line is short, at low frequency the magnetic field in magnetostatics is:

$$
B_{\theta}=\frac{\mu_{0} I}{4 \pi R} *(\cos (a)+\cos (b))
$$

To analyse the magnetic coupling between the test coil and the primary current, the following parameters are required

i. The magnetic field $B_{p}$ due 1amp current going through the plasma (or track or wire).

ii. The magnetic field $B_{t}$ at the centre of the test coil due to $1 \mathrm{amp}$ current flowing through itself. 

iii. The inductance of the plasma $L_{p}$
iv. The inductance of the test coil $L_{t}$

It was calculated using a magnetic field simulator that the magnetic coupling coefficient between primary current and the test coil is:

$$
K=\frac{B_{p}}{B_{t}} \sqrt{\frac{L_{t}}{L_{p}}}
$$

This coupling factor can be used to simulate the magnetic sensor in the proximity to the discharging current by using a simple spice application. In addition, the voltage at the test point of the Fig. 1 is calculated using the LTspice and it is given as

$$
V(\text { test })=I_{p} * K * \frac{\sqrt{L_{p} * L}}{R * C}
$$

Where $L_{p}$ is the inductance of the segment that generates the magnetic field and $L$ is the inductance of the sensor. This equation can be tested using LTspice simulation by varying the 4 coefficients: R, L, C and the coupling coefficient $K$. Therefore, simulation is a very important aspect in designing the proposed magnetic sensor and it is essential to determine the value of magnetic coupling coefficient. The LTspice codes are provided in the Annex to help the engineers to design, build and install this type of sensors.Alternatively, the sensor can be calibrated by sending a known current through the plasma and measuring the voltage at the test point. If a large current is observed a discharging capacitor with known capacity and voltage can be used. The current can be calculated by measuring the voltage drop at a sensing resistor installed serially with the capacitor. Also, it can be obtained by measuring the voltage drop at the capacitor during the discharge with aid of the relation:

$$
I=c * \frac{d V}{d t}
$$

\section{Equations used to measure Magnetic field \& pressure}

The open circuit voltage of a coil or loop can be obtained from faraday's law:

$$
V=-\frac{d \emptyset}{d t} * N=-A * N * \frac{d B}{d t}
$$

Using spice simulation file at Annex-2 it is checked:

$$
\text { Vtest }=-\frac{A * N}{R C} * B
$$

Where $\mathrm{N}$ is the number of turns in the sensor inductor and $\mathrm{A}$ is its area in squared meters. If any currents present are parallel to a magnetic field $\mathrm{j} X \mathrm{~B}=0$, then magnetic pressure can be obtained from magnetic field :

$$
\text { Pmag }=\frac{B^{2}}{2 \mu_{0}}
$$

\section{Non-stationary magnetic fields due a current going through a segment of wire or plasma}

In the field of electromagnetism, Jefimenko Eqs. [13-14] describe the behaviour of the magnetic fields in terms of the current distribution at retarded times. According to Jefimenko's equations, the magnetic field (B) outside of a cylindrical current flux due to a non-static current is given as follows.

$$
\begin{aligned}
& B(r, t)=-\frac{\mu_{0}}{4 \pi} f\left[\frac{r-r^{\prime}}{\left|r-r^{\prime}\right|^{3}} \times J\left(r^{\prime}, t_{r}\right)+\frac{r-r^{\prime}}{c\left|r-r^{\prime}\right|^{2}} \times\right. \\
& \left.\frac{\partial J\left(r^{\prime}, t_{r}\right)}{\partial t}\right] d^{3} r^{\prime}
\end{aligned}
$$

Where $r^{\prime}$ is a point in the charge distribution and $r$ is a point in space as shown in Fig. 8. $\mathrm{c}$ is the velocity of the light and $t_{r}$ is the delayed time. Since the electromagnetic information travels at the speed of light an observer at a distance from the charge distribution receive the information of earlier times and positions. Therefore, in case of non-static charge distribution the status of the source at some earlier time $t_{r}$ is important. The delay in traveling a distance $\left|r-r^{\prime}\right|$ is $\frac{\left|r-r^{\prime}\right|}{c}$. Hence, $t_{r}=t-\frac{\left|r-r^{\prime}\right|}{c}$

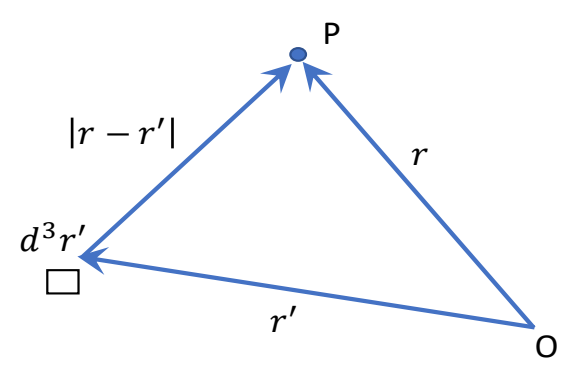

Figure 6: Displacement for retarded potential. 
If the distance from the centre of the loop to the observer point and the loop size is much less than the wavelength of the magnetic field, $t_{r}=t$. Thus, for a cylinder of plasma current or a current going through a cylinder wire, Eq. 12 can be written in the form

$$
B_{\theta}=-\frac{\mu_{0}}{4 \pi}\left[\int \frac{I * \bar{r} \times d \bar{z}}{r^{3}}+\int \frac{\bar{r} \times d \bar{z}}{c r^{2}} * \frac{d I}{d t}\right]
$$

At point $P$ (Fig. 7), the magnetic fields due to all current elements have the same direction. Thus, the net magnetic field can be calculated by evaluating the scalar sum of the contributions of the elements. With

$\bar{r} \times d \bar{z}=r \sin \theta d z$

The equation (13) can be written as

$B_{\theta}=-\frac{\mu_{0}}{4 \pi}\left[\int \frac{I * r \sin \theta}{r^{3}} d z-\frac{d I}{c d t} \int \frac{r \sin \theta}{r^{2}} d z\right]$

From Fig. 6 it can be seen that

$$
\begin{gathered}
\sin \theta=\frac{R}{r} \\
\cos \theta=\frac{z}{r} \\
d \bar{z}=r * d \theta \\
r=\sqrt{R^{2}+z^{2}}
\end{gathered}
$$

Substituting Eq. (15) into Eq. (14)

$$
\begin{aligned}
& B_{\theta}=\frac{\mu_{0}}{4 \pi}\left[\int-\frac{I * R d z}{r^{3}}+\frac{d I}{c d t} \int \sin \theta d \theta\right] \\
& B_{\theta}=\frac{\mu_{0}}{4 \pi}\left[-\frac{I}{R} * \frac{z}{r}+\frac{d I}{c d t} \cos \theta\right]_{\theta=a}^{\theta=\pi-b} \\
& B_{\theta}=\frac{\mu_{0}}{4 \pi}\left[-\frac{I}{R} * \cos \theta+\frac{d I}{c d t} \cos \theta\right]_{\theta=a}^{\theta=\pi-b} \\
& B_{\theta}=\frac{\mu_{0}}{4 \pi}\left[-\frac{I}{R}+\frac{d I}{c d t}\right](\cos (a)+\cos (b))
\end{aligned}
$$

\section{On axis non-stationary magnetic fields due a current loop}

The magnetic field at an observation point $P$ at the axis of a current loop where $z$ is the distance to the loop centre will be proportional to the current at the loop retarded a time that is $d / c$.



Figure 9: Displacement for retarded potential.

The integral at Eq. (13) can be written as:

$B_{z}=\frac{\mu_{0}}{4 \pi}\left[\int-\frac{I * \sin \theta d l}{d^{2}}+\frac{d I}{c d t} \int \frac{\sin \theta d l}{d}\right]$

As long as $\sin \theta=R / d$ and $d l=R * d \varphi$ :

$B_{z}=\frac{\mu_{0}}{4 \pi}\left[\int_{0}^{2 \pi}-\frac{I * R^{2} * d \varphi}{d^{3}}+\frac{d I}{c d t} \int_{0}^{2 \pi} \frac{R^{2} * d \varphi}{d^{2}}\right]$

$B_{z}=\frac{\mu_{0}}{4 \pi}\left[-\frac{I * R^{2} * 2 \pi}{d^{3}}+\frac{d I}{c d t} \frac{R^{2} * 2 \pi}{d^{2}}\right]$

$B_{Z}=\frac{\mu_{0}}{2}\left[-\frac{I * R^{2}}{d^{3}}+\frac{d I}{c d t} \frac{R^{2}}{d^{2}}\right]$

If the discharged current is a sine waveform $I(t)=10 * \sin (w t)$, then $d l / d t=10^{*} w^{*} \cos (w t)$, so the maximum time derivative current is $10^{*} \mathrm{~W}$. At that point the non-stationary magnetic current is larger than the stationary part when:

$$
\frac{w * d}{c}>1
$$

\section{Measuring the TOF}

The Time of Flight can be measured from the phase change in the plasma in the coil. When a plasma ball with a discharging current, pass over the coil as shown in Fig. 10 (it is a simplified 2dimension drawing), the current and voltage will experience a phase change of $180^{\circ}$ due to the changes of magnetic flux direction with respect to the coil. The measurement coil will detect both: the variation of the flux due variation of plasma current and due magnetic field increasing as 
plasma is nearer, so it will be detected the TOF if the variation of the current of the plasma is small or slow compared with the variation due plasma movement:

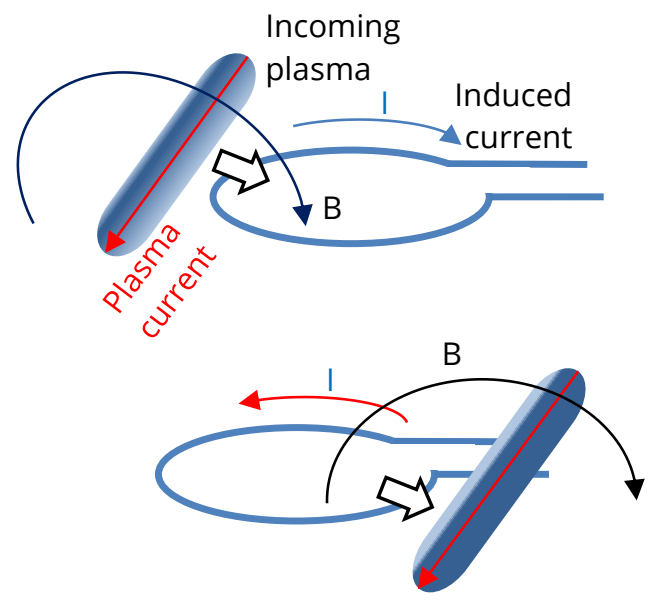

Figure $10.180^{\circ}$ phase change in the induced current in the coil due a coming plasma containing a current

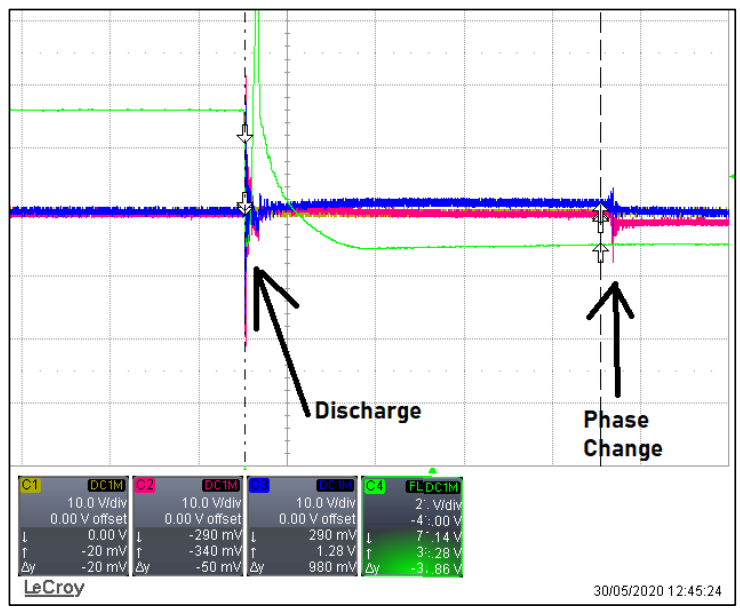

Figure 11 shows the scope plot of the test number 3042 obtained during the test in Pulstron-3. A phase change of $180^{\circ}$ in test point voltage and Fig. 12 shows the captured image of the Test 3042 where target and sensors are located just at the centre of the blast. The test sensor array was installed a few millimetres away from the target at several distances so as can be seen the sensors must withstand high energy discharge pulses.



Figure 12: Capture image of Test 3042 in Advanced Ignition SL.

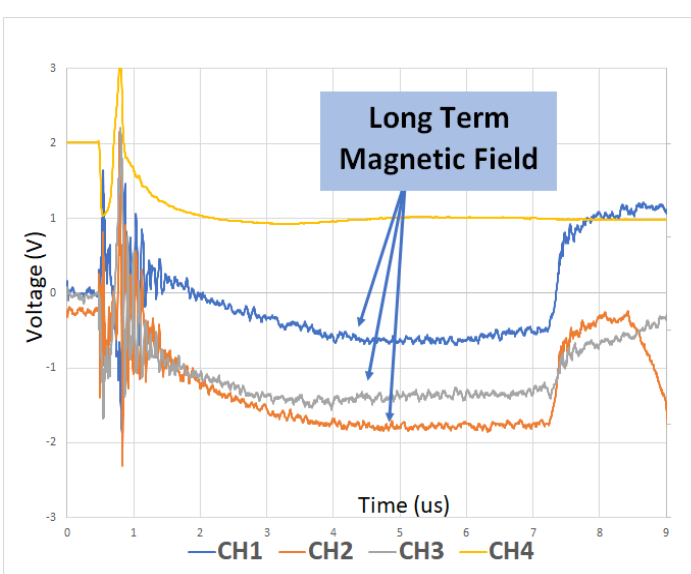

Figure 13: Magnetic field. Ch1 to 3 are 3 sensors in line and Ch4 is the discharging capacitor voltage

\section{Problem with transients and memory effect}

The proposed magnetic sensors were installed in the Pulsotron-3 Z-pinch machine and during the tests it was observed that sometimes a large magnetic field remains after the discharged current was finished, as can be seen in Fig. 13. It was investigated that due to a memory effect related to the fact that at the beginning of the test several extreme high frequency pulses appear which could be beyond the maximum range of the sensor. However, in the simulation no memory effect is observed which can be seen in the Fig. 14 and Fig. 15. Fig. 14 shows the injected pulsed current in the primary coil with different ramps slopes but with the same amplitude. Consequently, the higher frequency pulses obtained less voltage amplitude at the test point, but the end level was the same, as can be seen in the Fig. 15, then the remaining magnetic field observed at Fig.-13 corresponds to a longtime high energy magnetic field, perhaps due to a 
self-generating magnetic field in a rotating plasma or a breakdown voltage at sensor resistor

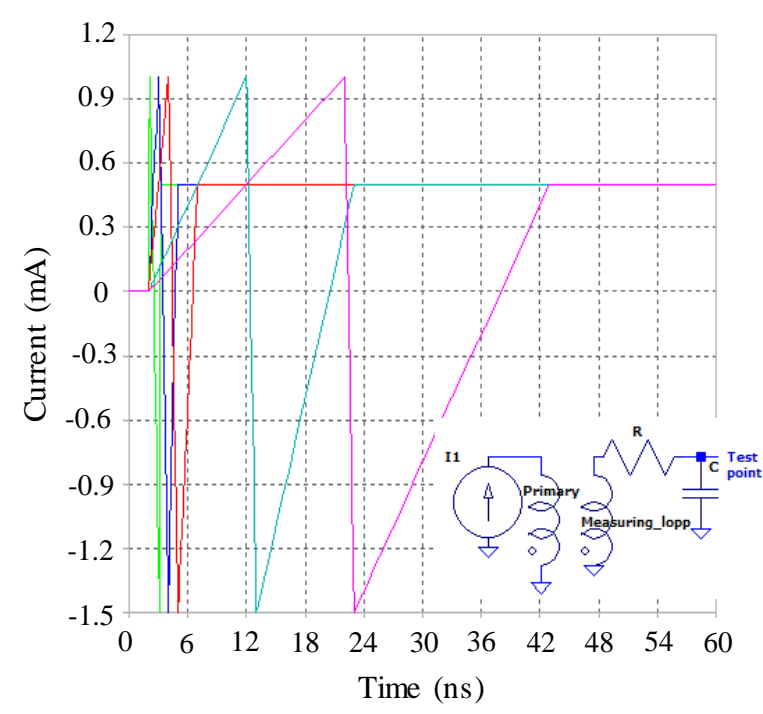

Figure 14: Injected 5 pulsed current at primary coil with different ramp slopes but same amplitude.

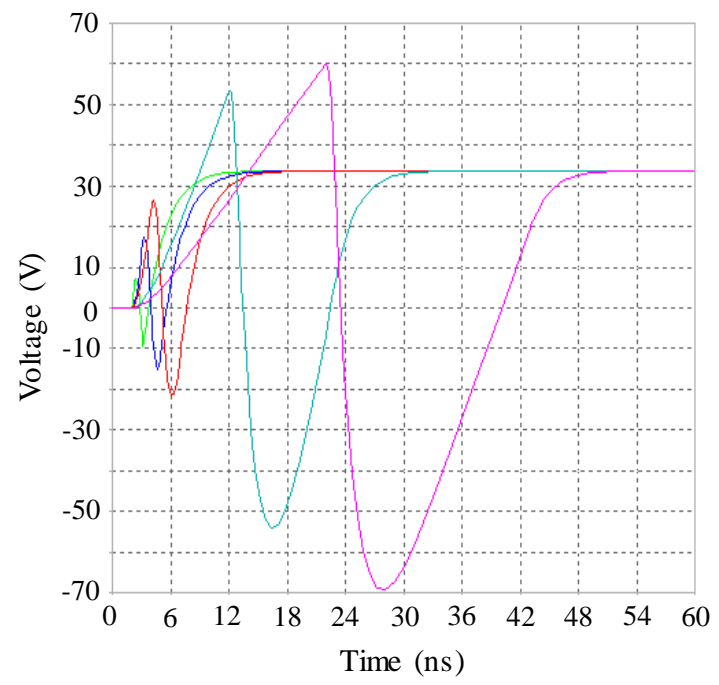

Figure 15: Out voltage at test point corresponding to the pulses with the same colour at Fig. 13.

\section{Conclusions}

In this paper, a magnetic sensor is presented which can be used to diagnose the magnetic field across a broadband frequency range. The values of the sensor component can be modified to operate in the desired frequency band and for different levels of magnetic fields. Several equations and LTspice simulation files are provided to help the engineers to design and build the sensor. In addition, the equations are provided for calculating non-stationary magnetic field due to rapidly varying current by using the sensor. The magnetic sensors are fabricated and installed in Pulsotron-3 Z-pinch machine. It is observed in simulations that there are no memory effect due previous high frequency pulses.

\section{References}

[1] T.F.R. Equipe, “Tokamak Plasma Diagnostics," Nucl. Fusion (1978), vol. 18, p. 647.

[2] I. H. Hutchinson, "Principles of Plasma Diagnostics", 2nd ed.; Cambridge University Press: Cambridge, UK, 2002; Chapter 2.

[3] V.D. Pustovitov, “Magnetic Diagnostics: General Principles and the Problem of Reconstruction of Plasma Current and Pressure Profiles in Toroidal Systems," Nucl. Fusion(2001), vol. 41, p. 721.

[4] E. J. Strait, " Magnetic diagnostic system of the DIII-D tokamak," Nucl. Fusion 2006, vol. 77, no. 2, 2006.

[5] C. Qing, L. Hong-bin H. Ben-xiong and D. Qiao-qi, "Rogowski sensor for plasma current," Sensor sJournal, IEEE, vol.9, no.3, pp. 293-296, March 2009.

[6] G. Grandi and M. Landini, "Magnetic-field transducer based on closed-loop operation of magnetic sensors," IEEE Transaction on Industrial Electronics, vol. 53, no. 3, pp. 880-885, Jun. 2006.

[7] H. A. Wheeler, "Inductance formulas for circular and square coils," Proceedings of the IEEE, vol. 70, no. 12, pp. 1449-1450, Dec. 1982.

[8] R. Edward, Hall-Effect Sensors. Elsevier, Elsevier, 2011. ISBN 978-0-7506-7934-3.

[9] L. C. Appel, M. J. Hole, "Calibration of the high-frequency magnetic fluctuation diagnostic in plasma devices," Rev. Sci. Instrum., Vol. 76, no. 9, 2005.

[10] S. Tumanski, "Induction coil sensors-A review," Meas. Sci. Technol., Vol. 18, no. 3, 19 Jan, 2007.

[11] LTspice, Design Center, Analog Devices, 2020, [Online]. Available: https://www.analog.com/en/designcenter/design-tools-and-calculators/tspice simulator.html. 
[12] J. C. Maxwell, Treatise on Electricity and Magnetism, Oxford. Vol. 2, part. 4, ch. 2 (pp. 502527) \& ch. 23 (pp. 845-866), 1904.

[13] O. D. Jefimenko, "Electricity and Magnetism: An Introduction to the Theory of Electric and Magnetic Fields", Appleton-CenturyCrofts (New-York - 1966). $2^{\text {nd }}$ ed.: Electret Scientific (Star City - 1989), ISBN 978-0-917406-089.

[14] David J. Griffiths, Mark A. Heald, "Timedependent generalizations of the Biot-Savart and Coulomb laws," American Journal of Physics 59 (2) (1991), 111-117.

\section{Annex-1: Simulation File 1}

This section contains the simulation file which can be used to simulate and build the magnetic sensor. The codes can be copied to file "Mag_sensor.asc" and simulated using LTspice ${ }^{\circledR}$ to check values explained in the present Paper.

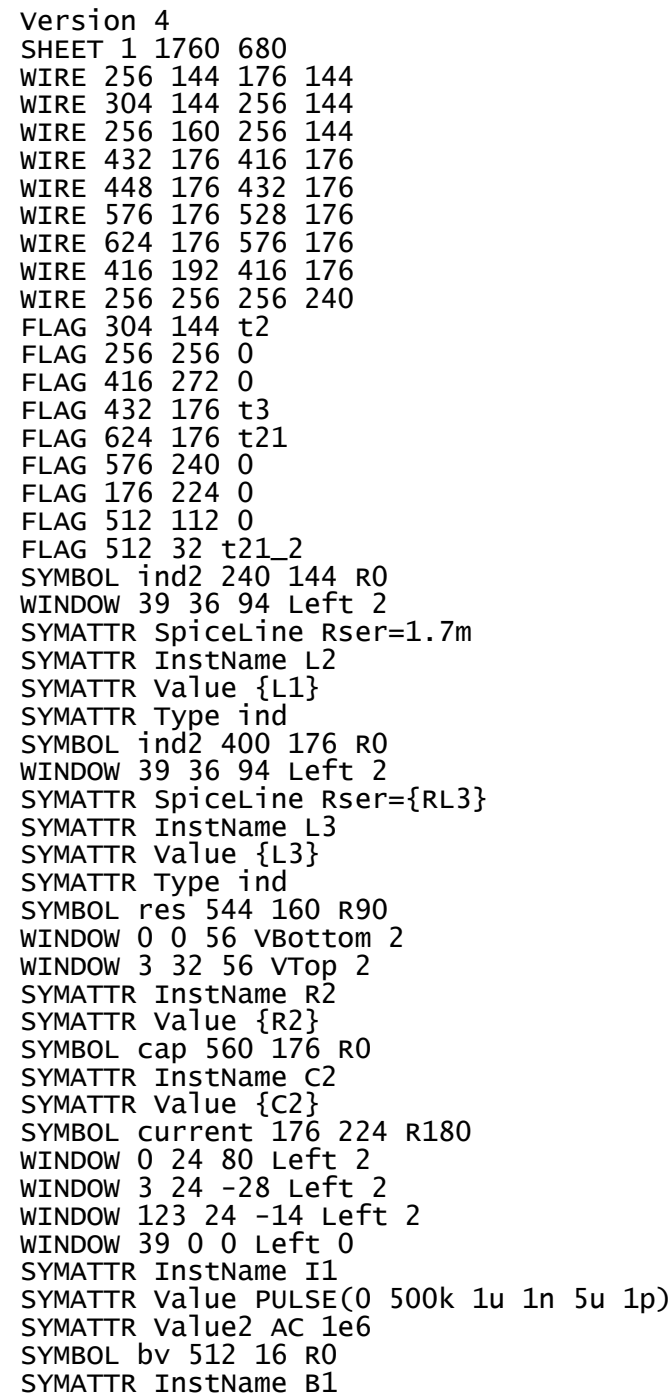

SYMATTR value $V=I(L 2) *\{K K 1\} * \operatorname{sqrt}(L 1 * L 3) /(R 2 * C 2)$

TEXT $96-8$ Left 2 ! param $L 3=5.64 n$ RL $3=1 p$

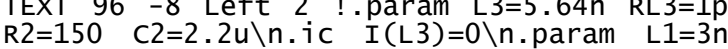
$\backslash n$. param KK1=0.0017 $\backslash n$.meas tran V1 $P P(V(t 21)) \backslash n$.MEAS AC Vmax max mag(V(t21)); find the peak response $\backslash n$.MEAS AC BW trig $\operatorname{mag}(\mathrm{V}(\mathrm{t} 21))=\mathrm{Vmax} / \mathrm{sqrt}(2) \quad \mathrm{rise}=1 \backslash \mathrm{n}+$ targ $\operatorname{mag}(\mathrm{V}(\mathrm{t} 21))=\mathrm{Vmax} / \operatorname{sqrt}(2)$ fall=1ast TEXT 360136 Left 2 ! K1 L2 L3 \{KK1\}

TEXT 104288 Left 2 !; ; tran 0 7u 0 $0.001 \mathrm{u} \backslash \mathrm{n}$.ac oct $50110100 \mathrm{GHz}$

TEXT 240 304 Left 2 ; Uncomment to check the Bandwidth

TEXT 52016 Left 2 ; This voltage must be equal to $t 21$

TEXT 240288 Left 2 ; Uncomment to check the $v(t 21)=V\left(t 21 \_2\right)$

\section{Annex-2: Simulation File 2}

This section contains the simulation file which can be used to simulate and build the magnetic sensor to measure the magnetic field and the magnetic pressure also.

The sensor inductor area is 10-4 squared meters and $N=1$. The codes can be copied to file "Mag_sensor-2.asc" and simulated using LTspice ${ }^{\circledR}$ to check values explained in the present Paper.

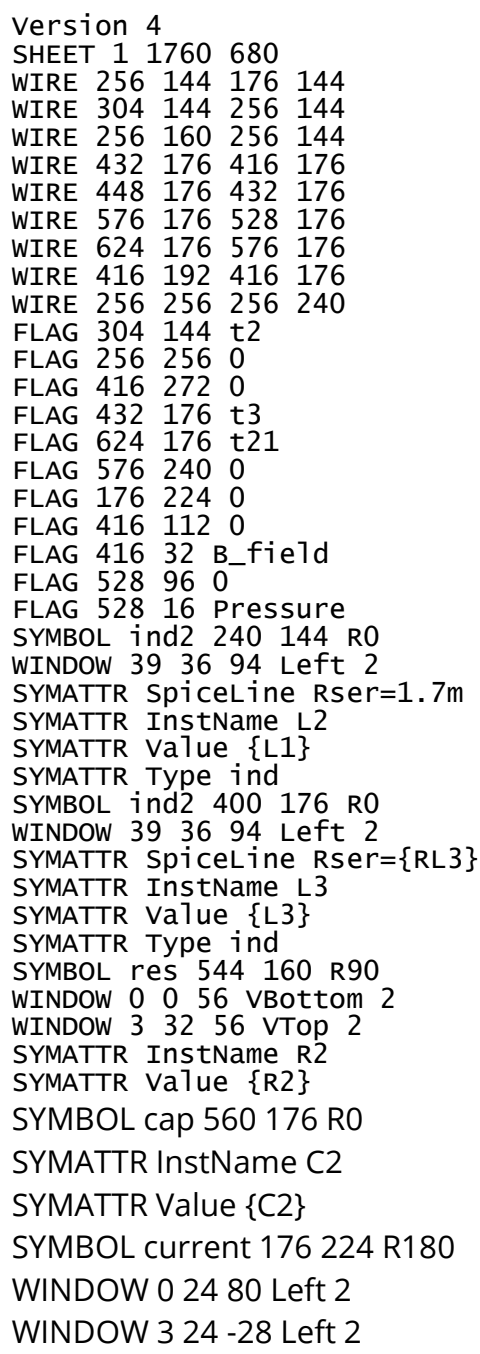


WINDOW 12324 -14 Left 2

WINDOW 3900 Left 0

SYMATTR InstName I1

SYMATTR Value SINE(0 \{500k/0.54772\} \{frec $\})$

SYMATTR Value2 AC 1 e6

SYMBOL bv 41616 RO

SYMATTR InstName B1

SYMATTR Value $\mathrm{V}=\mathrm{V}(\mathrm{t} 21) * \mathrm{R} 2{ }^{*} \mathrm{C} 2 /($ Area* $\mathrm{N})$

SYMBOL bv 5280 RO

WINDOW 33076 Left 2

SYMATTR InstName B2

SYMATTR Value $V=\left(V\left(B_{-}\right.\right.$field $\left.) * * 2\right) /(8 e-7 *$ pi $)$

TEXT -272 80 Left 2 !.param Area $=1 \mathrm{e}-4$ $N=1 \backslash n$.param $L 3=10 n R L 3=1 p \quad R 2=100 \quad C 2=1 u \backslash n$.ic $\mathrm{I}(\mathrm{L} 3)=0 \backslash n$.param $\quad \mathrm{L} 1=3 \mathrm{n} \quad$ In.param $\mathrm{KK} 1=0.01$ $\mathrm{W}=10 \mathrm{Mega}$ frec $=\mathrm{W} / 2 / \mathrm{PI} \backslash \mathrm{n}$.meas tran $\mathrm{V} 1$ $\mathrm{PP}(\mathrm{V}(\mathrm{t} 21)) \backslash \mathrm{n}$.meas tran $\mathrm{V} 3 \quad \mathrm{PP}(\mathrm{V}(\mathrm{t} 3)) \backslash \mathrm{n}$. MEAS AC Vmax max $\operatorname{mag}(\mathrm{V}(\mathrm{t} 21)) ;$ find the peak response $\backslash n . M E A S \quad A C \quad B W \quad$ trig $\operatorname{mag}(\mathrm{V}(\mathrm{t} 21))=\mathrm{Vmax} / \mathrm{sqrt}(2) \quad$ rise $=1 \backslash \mathrm{n}+\quad \operatorname{targ}$ $\operatorname{mag}(\mathrm{V}(\mathrm{t} 21))=\mathrm{Vmax} / \mathrm{sqrt}(2)$ fall=last $\backslash n$.meas tran Ipp $\operatorname{PP}(\mathrm{I}(\mathrm{L} 3))$

TEXT 360136 Left 2 !K1 L2 L3 \{KK1\}

TEXT 8288 Left 2 !.tran 0 \{5/frec $\} \quad\{1 /$ frec $\}$ $\{0.001 /$ frec $\} \backslash$ n;.ac oct $50110100 \mathrm{GHz}$

TEXT 240304 Left 2 ;Uncomment to check the Bandwidth

TEXT 240288 Left 2 ; Uncomment to check the $\mathrm{V}(\mathrm{t} 21)=\mathrm{V}\left(\mathrm{t} 21 \_2\right)$

\section{(ㄷ)(1)}

Open Access. This article is licensed under a Creative Commons Attribution 4.0 International License, which permits use, sharing, adaptation, distribution and reproduction in any medium or format, as long as you give appropriate credit to the original author(s) and the source, provide a link to the Creative Commons license, and indicate if changes were made. The images or other third party material in this article are included in the article $s$ Creative Commons license, unless indicated otherwise in a credit line to the material. If material is not included in the article's Creative Commons license and your intended use is not permitted by statutory regulation or exceeds the permitted use, you will need to obtain permission directly from the copyright holder. To view a copy of this license, visit: http://creativecommons.org/licenses/by/4.0/. 